\title{
Neural Masking by Sub-threshold Electric Stimuli: Animal and Computer Model Results
}

\author{
Charles A. Miller ${ }^{1,2}$, Jihwan Woo $^{3}$, Paul J. Abbas ${ }^{1,2}$, Ning Hu ${ }^{4}$, and Barbara K. Robinson ${ }^{1}$ \\ ${ }^{1}$ Department of Otolaryngology, University of Iowa Hospitals E Clinics, 21201 PFP, 200 Hawkins Drive, Iowa City, IA 52242, \\ USA \\ ${ }^{2}$ Department of Communication Sciences and Disorders, Wendell Johnson Speech and Hearing Center, The University of Iowa, \\ Iowa City, IA 52242, USA \\ ${ }^{3}$ Department of Biomedical Engineering, University of Ulsan, Ulsan, 680-749, South Korea \\ ${ }^{4}$ Hough Ear Institute, 3400 N.W. 56th Street, Oklahoma City, OK 73112, USA
}

Received: 29 April 2010; Accepted: 28 October 2010; Online publication: 16 November 2010

\begin{abstract}
Electric stimuli can prosthetically excite auditory nerve fibers to partially restore sensory function to individuals impaired by profound or severe hearing loss. While basic response properties of electrically stimulated auditory nerve fibers (ANF) are known, responses to complex, time-changing stimuli used clinically are inadequately understood. We report that forward-masker pulse trains can enhance and reduce ANF responsiveness to subsequent stimuli and the novel observation that sub-threshold (nonspike-evoking) electric trains can reduce responsiveness to subsequent pulse-train stimuli. The effect is observed in the responses of cat ANFs and shown by a computational biophysical ANF model that simulates rate adaptation through integration of external potassium cation (K) channels. Both low-threshold (i.e., Klt) and high-threshold (Kht) channels were simulated at each node of Ranvier. Model versions without Klt channels did not produce the sub-threshold effect. These results suggest that some such accumulation mechanism, along with Klt channels, may underlie sub-threshold masking observed in cat ANF responses. As multichannel auditory prostheses typically present sub-threshold stimuli to various ANF subsets, there is clear relevance of these findings to clinical situations.
\end{abstract}

Correspondence to: Paul J. Abbas · Department of Communication Sciences and Disorders, Wendell Johnson Speech and Hearing Center - The University of Iowa - Iowa City, IA 52242, USA. Telephone: 1-319-335-8733; fax: 335-8851; email: paul-abbas@uiowa.edu
Keywords: electric stimulation, auditory nerve, subthreshold, cochlear implant, electric pulse train, adaptation, single fiber, functional electric stimulation, computer model

\section{INTRODUCTION}

Auditory prostheses often use modulated pulse trains and multiple stimulus electrodes to excite sub-populations of auditory nerve fibers (ANFs) to partially restore the temporal and place coding of acoustically evoked ANF activity. Electric stimuli induce states of refractoriness and longer-term reduced excitability, typically referred to as adaptation. Sub-threshold stimuli, those that do not evoke action potentials, can lower ANF threshold through temporal integration at the membrane (Stypulkowski and van den Honert 1984; Hartmann et al. 1984; Dynes 1996; Cartee et al. 2000; Miller et al. 2001b). These linear and nonlinear effects are relevant to the designer of prosthetic devices, as they may limit information transfer and a user's perception of sound.

Cartee et al. (2000) reported that pulses presented $100-300 \mu$ s prior to a probe pulse increase ANF response probability, indicating stimulus integration. Dynes (1996) used sub-threshold multiple-pulse maskers $(1,4$, or 40 pulses presented at $1,000 \mathrm{pulse} / \mathrm{s})$ to explore their effects on subsequent probe pulses. Each reduced probe threshold, but the reductions 
became smaller as the number of masker pulses increased. A simple model of stimulus integration is inadequate to account for poorer integration with more masker pulses.

This report builds upon the background and studies of ANF responses to electric pulse trains (Litvak et al. 2003; Zhang et al. 2007; Miller et al. 2008) by adding a preceding masker train and determining its effect on responses to a subsequent probe train. Masker train durations were typically $300 \mathrm{~ms}$, comparable to speech segment durations. Masker-evoked spike activity reduced probe responses, with a relatively slow $(>200 \mathrm{~ms})$ recovery period. However, we found that maskers with levels insufficient to evoke spikes could cause forward masking. We developed a new version of our biophysical model of a cat ANF to explore membrane ionic mechanisms that might underlie sub-threshold masking. Following Woo et al. (2009a, b), it incorporated a leaky integrator based on $\mathrm{K}$ (cation) efflux, which provided a means of simulating spike-rate adaptation. ${ }^{1}$ In this report, model complexity was increased to include low- and high-threshold $\mathrm{K}$ channels (Klt and Kht channels, respectively) at each node. Previously only Kht channels were modeled.

For several years, the differing kinetics of Klt and Kht channels have been considered as a means of coding stimulus features by auditory brainstem neurons (Brew and Forsythe 1995; Bal and Ortel 2001). Also, a spatially organized heterogeneity of K currents and kinetics can exist across ANF populations (Mo and Davis 1997; Adamson et al. 2002; Liu and Davis 2007). Recently, Klt channels were productively used for biophysical modeling of rate adaptation for electrically stimulated fibers (Bruce and Negm 2009). Klt channels are also considered critical for modeling electrically evoked responses of myelinated fibers of nonauditory, neural tissue, such as mammalian motoneurons (McIntyre et al. 2002). This report includes cat data and results from a biophysical model with Kht and Klt channels, as well as a model variant without the Klt channels to assess the degree to which Klt channels may influence sub-threshold effects.

\section{METHODS}

\section{Animal model and preparation}

Adult cats free of visible middle-ear infection were used in acute recording sessions during which subjects were maintained at surgical levels of anesthesia, as

\footnotetext{
$\overline{1}$ Throughout this paper, potassium and other cations are indicated by their elemental abbreviation without the use of a superscript indicating positive charge. This was done for consistency with previous papers that discuss Klt and Kht channels and also for the sake of simplicity.
}

assessed by respiration pattern and pinch reflex. Other signs (heart rate, core temperature, pulse oximetry, and expired $\mathrm{CO}_{2}$ ) were monitored using a vital signs monitor (Model 400B, Pace Tech, Clearwater, FL, USA) and a capnometer (BCI Industries, Smiths Medical, St. Paul, MN, USA). Details of sedation, surgical anesthesia, and auditory nerve exposure are described elsewhere (Zhang et al. 2007). Other procedures were limited to tracheotomy for ventilation controlled by a Harvard Apparatus (Holliston, MA, USA) Model 665 ventilator, removal of the left pinna prior to obtaining click-evoked auditory brainstem responses (ABRs), and insertion of an intracochlear electrode array. Prior to insertion, the cochlea was deafened as described below. The left auditory nerve was accessed to record action potentials from the nerve trunk. The animal's core temperature was maintained by a circulating warm-water jacket and insulating pads. Procedures were approved by the University of Iowa Animal Care and Use Committee and adhered to US NIH regulations.

Click-evoked ABRs were obtained from each subject before and after intracochlear administration of Neomycin sulfate $(50-60 \mu \mathrm{l})$ to assure that ABR threshold increased by at least $80 \mathrm{~dB}$. After opening the round window, the aminoglycoside was introduced as several (eight to ten) small boli, each followed by aspiration to cause a net increase in Neomycin concentration. This was done to eliminate or greatly reduce electrophonic responses (mediated by viable hair cells) that could confound analysis of spike activity caused by direct membrane depolarization.

\section{Electric stimuli}

After Neomycin infusion, an eight-electrode "banded electrode" array (P/N Z60274, Cochlear Ltd, Australia) was inserted into the scala tympani through the round window to a 5-5.5-mm depth. Only the most apical electrode of the array was used for the monopolar electrical stimulation employed for all experiments. The return current path was provided by a needle electrode inserted into neck muscle. Three techniques were used to prevent passage of direct current to the nerve tissue: (1) the use of symmetric biphasic current pulses, (2) the use of an optically isolated battery-powered current source, and (3) capacitive coupling of the current source output. All electric stimuli were composed of $40 \mu \mathrm{s} /$ phase symmetric biphasic rectangular pulses, with a leading cathodic phase. Search stimuli consisted of low-rate (30 pulse/s) pulse trains.

Experimental maskers and probes were pulse trains, each typically having $300 \mathrm{~ms}$ train durations, unless noted otherwise. A post probe-train silent 
interval of $1,200 \mathrm{~ms}$ was used to facilitate neural recovery. Two masker pulse rates (250 and 5,000 pulse/s) were systematically varied, and masker current levels were varied so that probe responses were obtained for sub-threshold maskers (i.e., too low to elicit any spikes) and rates approaching maximal (saturation) rates. Probe pulses were presented at 100 pulse/s, a compromise between high rates for good temporal resolution and low rates that avoid cumulative stimulus-evoked effects. Once a fiber was encountered, probe level was fixed to achieve a midrange firing efficiency (FE), typically between $30 \%$ and $70 \%$. Probe-alone FEs at or near $100 \%$ FE were avoided so that FE and probe-level changes would be correlated. Masker level was then varied to obtain a range of masking effects. Finally, when fiber contact time permitted, a series of post-stimulus-time histograms (PSTHs) were collected for multiple probe levels, so as to explore the effect of the magnitude of the probe response.

Probe-train onset followed the masker offset by a delay equal to one masker interpulse interval. Thus, for 5,000 pulse/s maskers, a 0.12-ms delay separated last-masker-pulse offset and first-probe-pulse onset. For 250 pulse/s maskers, a 3.92-ms delay separated masker offset and probe onset. These different masker-off/probe-on delays require consideration when short-lived phenomena, which could be missed by the existence of this 3.92-ms gap, occur. Specifically, with the use of single-pulse maskers and probes, integration of masker and probe energy is observed for short (0.1-0.4 ms) MPIs (Stypulkowski and van den Honert 1984; Dynes 1996; Cartee et al. 2000). Our paradigm would not capture this effect for relatively slow, 250 pulse/s, masker trains.

A probe train with no preceding masker was interleaved between each masker/probe/silentperiod combination to provide baseline "probe alone" response data. Interleaving reduced the impact of possible cumulative effects across repeated presentations (typically 30 or more repetitions, or "sweeps"). The probe-alone stimulus was identical to the masker/probe/silent-period stimulus, but with zero masker current, providing more than 1,200 ms for recovery from each masker/probe combination. The collection of one data set (yielding one masked and unmasked PSTH) required about $2 \mathrm{~min}$.

\section{Recording and analysis of feline data}

ANF spikes were recorded using a glass micropipette formed from borosilicate glass $(0.58 \mathrm{~mm}$ I.D., $1.0 \mathrm{~mm}$ O.D.) and filled with $0.15 \mathrm{M} \mathrm{KCl}$ in $0.05 \mathrm{M}$ Tris buffer. Potentials were amplified $(10 \times)$ and low-pass filtered (first-order, 10-kHz half-power frequency) by an Axon Instruments Axoprobe amplifier. Additional filtering was provided by a Butterworth high-pass filter (second order, $100-\mathrm{Hz}$ cutoff) and a Butterworth low-pass filter (sixth order, $30-\mathrm{kHz}$ cutoff). Electrical artifacts associated with the a.c. power line were reduced by a HumBug noise template subtraction circuit (Quest Scientific Instruments, North Vancouver, BC, Canada). The filtered potentials were digitally sampled at 100,000 samples/s using an Instrutech ITC-18 data acquisition board (HEKA Instruments, Bellmore, NY, USA) and stored for detailed off-line analysis using Matlab (The MathWorks, Natick, MA, USA) routines written in house. Spikes were extracted from stimulus artifacts using either "template subtraction" or boxcar filtering techniques, as described in Miller et al. (2008).

Responses to the masker and the probe trains were characterized by computing the overall spike rate or count occurring during the presentation of each train. Rates were computed as the average measured over repeated presentations. The spike analysis windows were approximately equal to the duration of the respective masker or probe train. For the maskeralone and probe train conditions, the analysis window included a 2-ms epoch after offset of the last masker pulse to capture any late spikes. The effect of a masker train on a subsequent probe train is reported as the "probe response recovery ratio", i.e., the ratio of probe spikes with preceding masking and probe spikes without a preceding masker. As a masked probe response recovers from adaptation, this ratio will typically increase from 0 (in cases with complete adaptation) and approach 1, indicating a response equal to the unmasked probe response.

\section{Computational model: general description}

The ANF model is a compartmental type that includes a cell body and peripheral and central axons, using known anatomical features of a cat ANFs (Arnesen and Osen 1978; Liberman and Oliver 1984; Woo et al. 2009a). Specifically, the bipolar neuron has four nodes on the peripheral axon and 23 nodes on the central axon. Internodal distances vary from 150 to $350 \mu \mathrm{m}$ according to reported anatomy. The cell body has a $300-\mu \mathrm{m}^{2}$ cross-sectional area and an elliptical shape of a rotationally symmetric solid with a 0.6 axis ratio. Peripheral fiber diameter, central fiber diameter, and nodal gap width were set at $1.2,2.3$, and $1 \mu \mathrm{m}$, respectively. The ratio of myelin thickness of the cell body to that of the central axon was measured by examining photomicrographs of fibers and cell bodies available from our histological sections. The length of the unmyelinated peripheral terminal was set at $10 \mu \mathrm{m}$ to produce experimentally realistic strengthduration curves (Colombo and Parkins 1987). Part of the development of the model was reported in Woo et 
al. (2009a). Parameters describing the kinetics of the $\mathrm{Na}$, Kht, and Klt channels are provided in Table 1.

\section{Membrane properties and nodal currents}

Each myelinated section is modeled by nine consecutive passive internodal compartments followed by an active node, which exclusively includes voltagedependent $\mathrm{Na}$ and $\mathrm{K}$ channels. The on-off states of these channels were approximated by a Markov jumping process, and their stochastic properties were simulated by a channel number tracking algorithm (Chow and White 1996; Mino et al. 2002). The transmembrane potential $V_{\mathrm{m}}$ resulting from the applied electric stimulus $V_{\mathrm{e}}$ in each axon compartment $[k]$ is described by Mino et al. (2004) and shown in Eq. 1 using discrete time steps:

$$
\begin{gathered}
-\left(\frac{V_{\mathrm{m}}^{[k+1]}[t]-V_{\mathrm{m}}^{[k]}[t]}{R_{\mathrm{a}}^{[k+1, k]}}-\frac{V_{\mathrm{m}}^{[k]}[t]-V_{\mathrm{m}}^{[k-1]}[t]}{R_{\mathrm{a}}^{[k, k-1]}}\right) \\
+C_{\mathrm{m}}^{[k]} \frac{V_{\mathrm{m}}^{[k]}[t+\Delta t]-V_{\mathrm{m}}^{[k]}[t]}{\Delta t}+\frac{V_{\mathrm{m}}^{[k]}[t]}{R_{\mathrm{m}}^{[k]}}+I_{\mathrm{ion}}^{[k]}[t] \\
=\frac{V_{\mathrm{e}}^{[k+1]}[t]-V_{\mathrm{e}}^{[k]}[t]}{R_{\mathrm{a}}^{[k+1, k]}}-\frac{V_{\mathrm{e}}^{[k]}[t]-V_{\mathrm{e}}^{[k-1]}[t]}{R_{\mathrm{a}}^{[k, k-1]}}
\end{gathered}
$$

The transmembrane potential in each axon compartment was determined by solving a partial differential equation using the Crank-Nicholson method (Mino et al. 2004). The values of the voltage-independent nodal resistance, $R_{\mathrm{m}}$, and nodal capacitance, $C_{\mathrm{m}}$, are the same as those reported in Woo et al. (2009b). Axoplasmic resistance, $R_{\mathrm{a}}$, internodal resistance, $r_{\mathrm{m}}$, internodal capacitance, $c_{\mathrm{m}}$, and ionic electrophysiological properties (channel density, $\rho$, and conductance, $\gamma$ ) were based on Mino et al. (2004) and Woo et al. (2009a).
Ion current $\left(I_{\text {ion }}\right)$ in each $k$-th active node at time $t$ was calculated by summing $\mathrm{Na}$ current $\left(I_{\mathrm{Na}}\right)$, Kht current $\left(I_{\text {Kht }}\right)$, and Klt current $\left(I_{\text {Klt }} ;\right.$ Krylov and Makovsky 1978; Schwarz et al. 1995; Imennov and Rubinstein 2009). Currents are described by equation set 2:

$$
\begin{aligned}
I_{\mathrm{Na}}^{[k]} & =\gamma_{\mathrm{Na}} N_{\mathrm{m} 3 h 1}(t, k)\left(V(t, k)-E_{\mathrm{Na}}(t, k)\right) \\
I_{\mathrm{Kht}}^{[k]} & =\gamma_{\mathrm{Kht}} N_{\mathrm{Kht}}(t, k)\left(V(t, k)-E_{\mathrm{Kht}}(t, k)\right) \\
I_{\mathrm{Klt}}^{[k]} & =\gamma_{\mathrm{Klt}} N_{\mathrm{Klt}}(t, k)\left(V-E_{\mathrm{Klt}}(t, k)\right)
\end{aligned}
$$

The ion channel conductances are $\gamma_{\mathrm{Na}}=22.65 \mathrm{pS}$, $\gamma_{\mathrm{Kht}}=20 \mathrm{pS}$, and $\gamma_{\mathrm{Klt}}=20 \mathrm{pS}$. The terms $N_{\mathrm{m} 3 \mathrm{~h} 1}, N_{\mathrm{Kht}}$, and $N_{\mathrm{Klt}}$ represent the number of activated channels for each channel type. Each channel type was assigned to the following nodal densities: $112 / \mu \mathrm{m}^{2}$ (Na), $10 / \mu \mathrm{m}^{2}$ (Kht), and $10 / \mu \mathrm{m}^{2}$ (Klt). The Klt channel Nernst potential, $E_{\mathrm{Kl}}$, was set to $-88 \mathrm{mV}$, equal to that of the Kht channel. $I_{\mathrm{Klt}}$ was calculated using a two-state (one open, $S_{1}$, one closed state, $S_{0}$ ) channel model. Opening $(\alpha)$ and closing $(\beta)$ rates were modified for mammalian temperature of $37^{\circ} \mathrm{C}$, as shown in Table 1.

\section{Model of rate adaptation}

The model incorporates a "leaky integrator" K cation storage mechanism that alters membrane voltage and simulates spike-rate adaptation (Woo et al. 2009a). External $\mathrm{K}$ concentration, $\left[K_{\text {ext }}\right]$, was governed by a

\begin{tabular}{|c|c|c|c|c|}
\hline \multicolumn{5}{|c|}{ Gating kinetics for the ANF model } \\
\hline \multirow{2}{*}{$\frac{\text { Gating kinetics }^{\text {a) }}}{\mathrm{Na}}$} & \multicolumn{2}{|l|}{ Value } & & Reference \\
\hline & A & $\mathrm{B}$ & $\mathrm{C}$ & \\
\hline$\alpha_{\mathrm{m}}$ & 1.872 & 25.41 & 6.06 & \\
\hline$\beta_{\mathrm{m}}$ & -3.793 & 21.001 & 9.41 & \\
\hline$\alpha_{\mathrm{h}}$ & -0.549 & -27.74 & 9.06 & \\
\hline$\beta_{\mathrm{h}}$ & 22.57 & 56 & 12.5 & Schwarz and Eikhof 1987; Matsuoka et al. 2001 \\
\hline \multicolumn{5}{|r|}{ 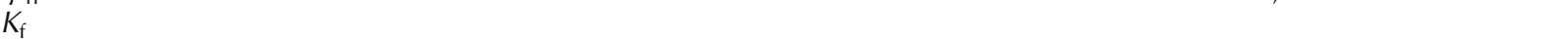 } \\
\hline$\alpha_{\mathrm{n}}$ & 0.129 & 35 & 10 & \\
\hline $\begin{array}{c}\beta_{\mathrm{n}} \\
K_{\mathrm{s}}^{\mathrm{b})}\end{array}$ & -0.324 & 35 & 10 & \\
\hline$\alpha_{\mathrm{s}}$ & 0.00789 & -100.5 & 23.6 & Schwarz et al. 1995 \\
\hline$\beta_{\mathrm{s}}$ & -0.00473 & 168.1 & -21.8 & \\
\hline
\end{tabular}
time-changing $\Delta\left[K_{\text {ext }}\right]$ value that depended upon recent $\mathrm{K}$ current, as indicated in the discrete time Eq. 3:

$$
\begin{aligned}
\Delta\left[K_{\text {ext }}\right]\left(N, n_{\mathrm{R}}\right) & =\sum_{i=0}^{N} I_{\mathrm{K}}\left(i, n_{\mathrm{R}}\right) \times g(N \Delta t-i \Delta t) \\
g(t) & =A_{\mathrm{f}} \times \exp \left(-t / \tau_{\text {depl }}\right)
\end{aligned}
$$

\section{TABLE 1}

${ }^{a)}$ Each gating kinetic was calculated based on following equation: $\alpha_{\mathrm{m}}, \alpha_{\mathrm{n}}, \alpha_{\mathrm{h}}, \alpha_{\mathrm{s}}, \beta_{\mathrm{m}}, \beta_{\mathrm{n}}, \beta_{\mathrm{s}}=A(V-B) /\{1-\exp [(B-V) / C]\}, \beta_{\mathrm{h}}=A /[1+\exp \{(B-V) / C\}]$

${ }^{\text {b) }}$ The values were temperature adjusted to $37^{\circ} \mathrm{C}$ using $\mathrm{Q}_{10}=3.0$. 
where $i$ is the time increment variable, $N$ is the final (or latest) time increment, $n_{\mathrm{R}}$ is $n$-th Ranvier node, $I_{\mathrm{K}}$ is K current, and $g(t)$ is an exponential decay function with amplitude $A_{\mathrm{f}}$ and time constant $\tau_{\text {depl }}$. [ $\left[K_{\text {ext }}\right]$ alteration also required updating both $E_{\mathrm{K}}$ and $E_{\text {rest }}$ using the Nernst and the Goldman-Hodgkin-Katz equations. This mechanism was first described in Woo et al. (2009a) and refined in Woo et al. (2009b).

\section{Simulations, model variants, and optimization}

Computations were performed on a desktop computer using Matlab 7.0 R12.1 (Mathworks, Inc., USA), with simulations using a $1-\mu$ s time step size. The simulations used a spherical $(0.45 \mathrm{~mm}$ diameter $)$ monopolar electrode positioned directly above the ninth active node (as counted from the peripheral process). External space was filled with an isotropic conducting medium with $0.3 \mathrm{k} \Omega / \mathrm{mm}$ resistivity (McNeal 1976). A high (5,000 pulse/s) rate pulse train (40 $\mathrm{s} /$ phase biphasic, cathodic polarity first) was delivered as a forward masker, followed by a lowrate (100 pulse/s) probe train for assessment of masking recovery. The modeled stimulus electrode was set over the fifth central node of Ranvier (or the ninth node, counting from the peripheral process). This position is shown in Figure 2B of Woo et al. (2010). Electrode-to-fiber distance was set at $1.225 \mathrm{~mm}$, measured from the center of the electrode to the membrane surface. Membrane potentials were recorded at the 16th central node of Ranvier. After each presentation of masker, probe, or masker + probe combination, the model parameters were reset and random variables were reseeded to new values so that the model would not create across-presentation cumulative effects.

Two different model versions were used so as to explore the influence of the Klt channels, a relatively novel feature of ANF computational models. What is referred to as the "full model" included Kht and Klt channels, whereas the simpler model only included Kht channels (Woo et al. 2009a). By comparing the predictions of each model, we could assess the likelihood that sub-threshold masking effects (observed in cat ANFs) involved Klt channel activity.

Our previous biophysical models (Woo et al. 2009a, b, 2010) simulated $\mathrm{Na}$ and Kht channels (but without Klt channels) and did not reproduce the sub-threshold masking observed in the cat ANF data. This led us to the addition of Klt channels to our previous model (Woo et al. 2009b) that simulated rate adaptation using the aforementioned external accumulation of $\mathrm{K}$ cations. The addition of the Klt channels required reoptimization of the model so that it would again approximate the response properties of real ANFs, such as conduction velocity, refractoriness, and rate adaptation (Miller et al. 2001b, 2004; Zhang et al. 2007). During the optimization process, we varied single channel conductivity $(\gamma)$ and density $(\rho)$ of both Klt and Kht channels from 10 to $40 \mathrm{pS}$ and from 10 to $50 / \mathrm{mm}^{2}$, respectively (Schwarz et al. 1995; Imennov and Rubinstein 2009). The ratio of the densities of Klt and Kht channels was set to either $1: 1$ or $2: 1$. For the full model, parameters that yielded responses similar to cat ANFs were found to be as follows: $\gamma_{\mathrm{Kht}}=20 \mathrm{pS}, \gamma_{\mathrm{Klt}}=20 \mathrm{pS}, \rho_{\mathrm{Kht}}=10 / \mathrm{mm}^{2}$, and $\rho_{\mathrm{Klt}}=10 / \mathrm{mm}^{2}$. Parameters of our K-based adaptation model (Woo et al. 2009b) were modified to account for the aforementioned parameter changes. Specifically, we adopted a new amplitude coefficient $\left(A_{\mathrm{f}}=\right.$ $0.117)$ and depletion time constant $\left(\tau_{\mathrm{dep}}=160 \mathrm{~ms}\right)$.

For model simulations of forward-masking effects of cat ANFs, the same two masker rates (250, $5,000 \mathrm{pulse} / \mathrm{s}$ ) were employed, as was the same probe pulse rate (100 pulse/s). Masker train and probe train duration was 200 and $250 \mathrm{~ms}$, respectively. These latter two values are smaller than those used for the cat ANF studies and were chosen as an expedient to reduce the large computation times required for each simulation. Probe levels were selected so that responses were in the upper part of the modeled fiber's dynamic range (i.e., FEs between $80 \%$ and $95 \%$ ).

\section{RESULTS}

\section{Exemplar cat and model ANF data}

In Figure 1, exemplar responses from a cat ANF (left columns) and the "full model" (with Klt and Kht channels) responses (right columns) are shown for a level series of 5,000 pulse/s maskers and fixed probetrain levels. Both the cat ANF and model PSTHs demonstrate sub-threshold masking. In these cases, the maskers were 200-ms-long, 5,000-pulse/s trains, and the probes were 250-ms-long, 100-pulse/s pulses trains. The probe-train PSTHs include responses to forwardmasked (black bars) and unmasked (gray bars) probes, with probe onset occurring $0.2 \mathrm{~ms}$ after masker offset. The first (left-most) column shows PSTHs from a cat ANF stimulated by the masker train. The top two masker PSTHs (rows A and B) show initially large rate alternations due to refractory effects (Javel 1990) and a rapid overall rate decrease typically attributed to "adaptation" (Zhang et al. 2007). The masker PSTH of row $\mathrm{C}$ shows a minimal (near threshold) response, while lower masker levels (rows D-F) were sub-threshold. Probe responses A through D clearly show rate suppression. For probe PSTHs for the two lowest masker levels (cases E and F), Bonferroni-corrected $t$ tests (accounting for the inclusion of PSTH combinations at six masker levels) indicated that the sub-threshold maskers 


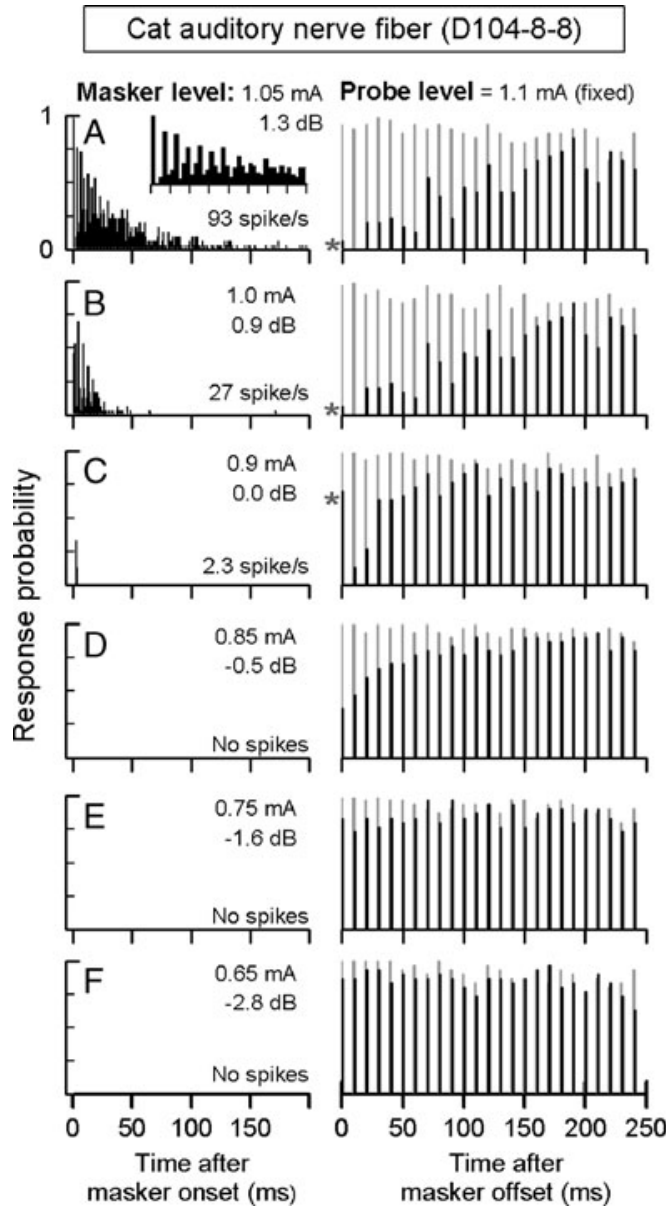

FIG. 1. Both the PSTHs from a cat ANF (left 2 columns) and the two $\mathrm{K}$ channel biophysical model (right 2 columns) exhibit sub-threshold masking of probe responses. In all cases, the masker was a 200-ms, 5,000-pulse/s train, and the probe was a 250-ms, 100-pulse/s train. Probe level is fixed, while masker level varies along each column. Masker levels are stated in milliamperes and decibels relative to the lowest masker level that evoked spikes. Shown near each masker PSTH is the mean overall spike rate. Masked probe responses are plotted in black; unmasked probe responses (the control condition)

reduced responses to the probe (worst case: $t=4.32$, $\left.p_{\text {error,corrected }}=0.0004, d f=25\right)$. In rows $\mathrm{A}-\mathrm{C}$, the forwardmasked response to the first probe pulse is elevated relative to that of the second pulse (asterisks), consistent with integration of masker and probe energy. Thus, preceding maskers can either increase or decrease responsiveness to pulses within a probe stimulus.

Model-generated PSTHs are shown in the third and fourth columns of Figure 1 for comparable effective levels of maskers and a fixed-level probe. Compared with the ANF data, the model PSTHs to supra-threshold maskers show similar onset responses followed by rate decays that are produced by the model's adaptation component. After presentation of supra-threshold maskers (rows G-I), probe responses are reduced and undergo recovery. While the three lowest maskers (J-L) evoked no spikes, the probe PSTHs in two of three cases ( $\mathrm{J}$ and $\mathrm{K}$ ) suggest sub-

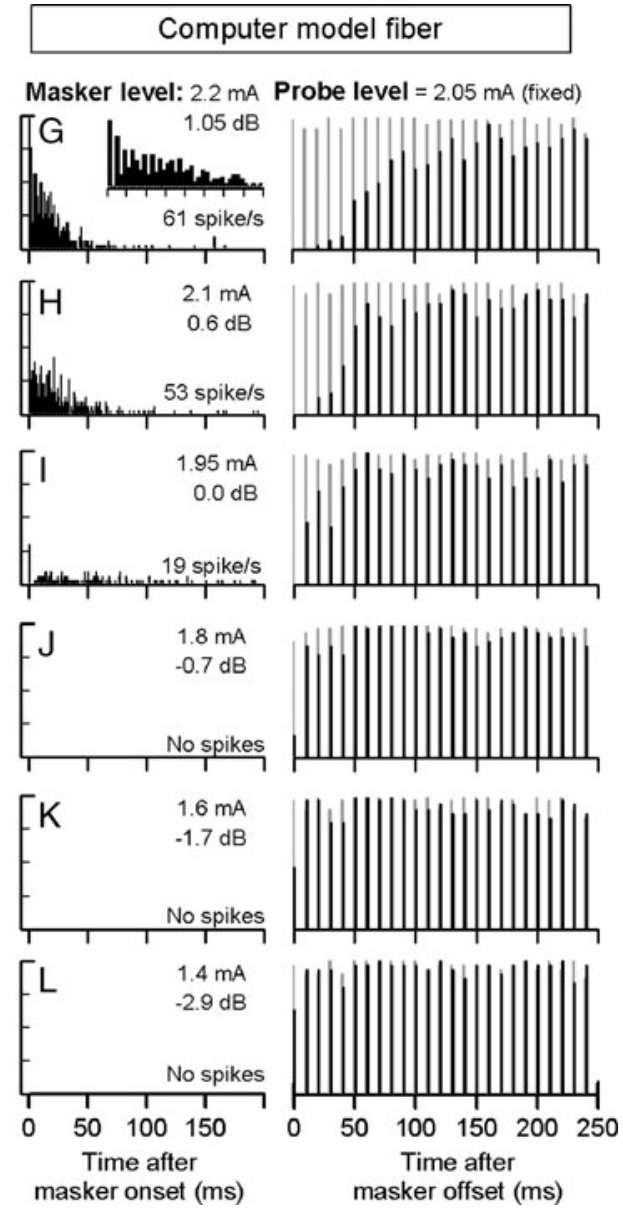

are in gray. Cat PSTHs for the probe stimuli show response reductions for both supra-threshold and sub-threshold maskers. At higher masker levels $(A-C)$, probe response recovery was nonmonotonic, with larger first-pulse responses (asterisks). Sub-threshold masking is also seen in $J$ and $K$ of the computer model PSTHs. All ordinate axes have ranges from 0 to 1 . The inset graphs in $A$ and $G$ show the details of the first $40 \mathrm{~ms}$ of each PSTH to better illustrate response probability alternations.

threshold effects. Bonferroni-corrected paired $t$ tests run on those three cases (i.e., data of panels $\mathrm{J}-\mathrm{L}$ ) showed significant sub-threshold masking in two of the cases $\left(\mathrm{J}\right.$ and $\mathrm{K}$; worst case $T=2.76, p_{\text {error,corrected }}=$ $0.033, d f=24)$. As in the case of the real cat data, the model PSTH could exhibit alternations in response probability to the high-rate masker (row G and inset PSTH). The alternation rate for the case shown is faster than what is observed from the cat fiber (row A). Finally, the model typically did not demonstrate increased responses to the first probe pulses that we attribute to integration of sub-threshold masker energy.

\section{Group trends}

Group analyses of cat data (Fig. 2 A, B) show robust sub-threshold masking that is dependent on masker 


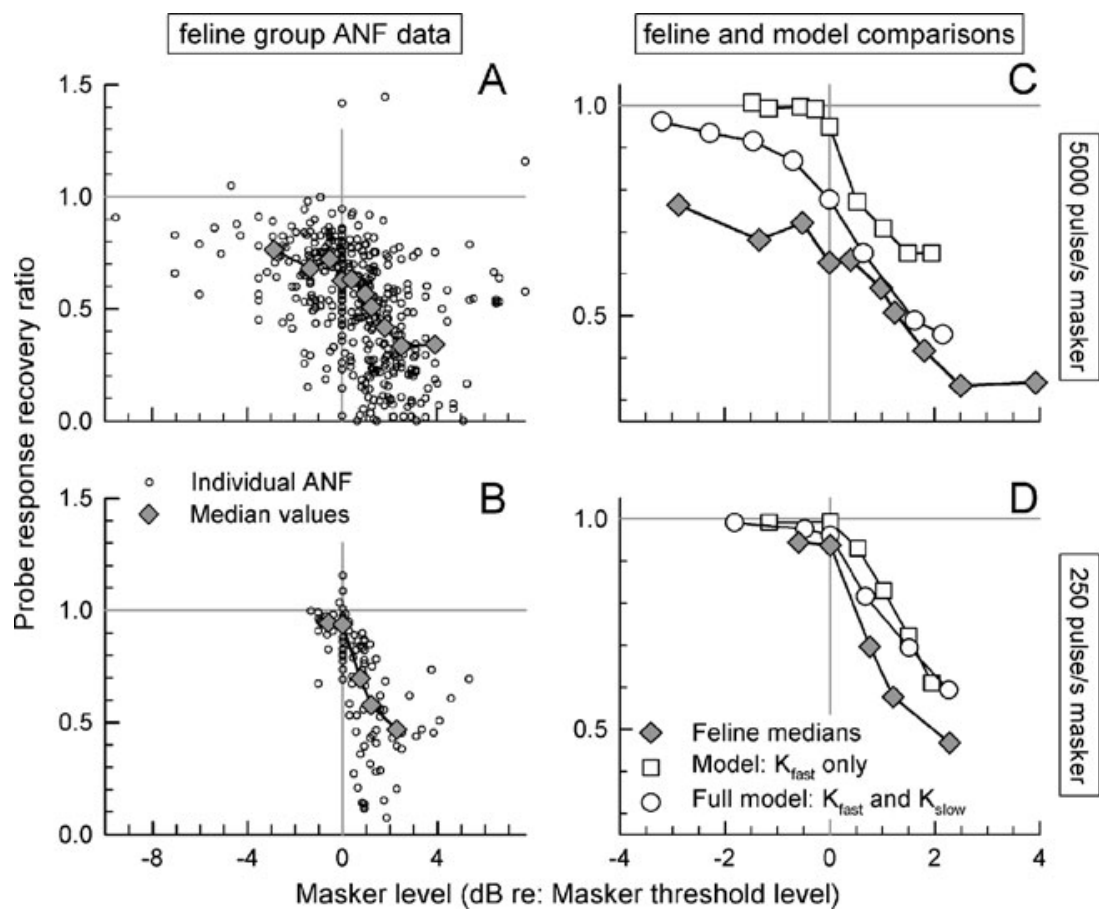

FIG. 2. The degree of forward masking of cat ANF responses to probe trains depends on masker level and masker pulse rate. Individual cat ANF recovery ratios are plotted in the graphs of the left column using small circles. Median values based on equal sample sizes are plotted using gray diamond symbols. The ratios are plotted versus effective masker level, with levels referenced to the lowest level that evoked spikes on a per-fiber basis. Probe responses are shown for 5,000 pulse/s maskers (upper graphs) and for 250 pulse/s maskers (lower graphs). Median values indicate that probe suppression is proportional to masker effective level and is greater for $5,000 \mathrm{pulse} / \mathrm{s}$ maskers. Feline medians are replotted in $C$ and $D$, along with results from computer model variants: the "full model" that uses both Klt and Kht channels (circles) and the model lacking Klt channels (squares). level and pulse rate. The abscissa ("Masker level") is defined relative to the lowest masker level that evoked spikes for each ANF. The ordinate is the ratio of the number of spikes evoked by masked and unmasked probe trains. Individual ANF data are plotted with circles, while diamonds indicate medians of subsets chosen along the abscissa dimension to have comparable numbers of observations. Probe recovery ratios are shown for high- and low-rate masking in Figure 2 A, B, respectively. In all cases, 300-ms maskers and probes were used. For 5,000 pulse/s masking, 48 ANFs contributed 417 points, while 22 of those ANFs contributed 108 data for 250 pulse/s masking. ANF data are combined in order to assess dominant trends; Effects of changing the response rate to the masker and to the probe are explored below.

For both masker rates, the recovery of the probe responses depended on masker level. For 5,000 pulse/s masking, all but two of 125 sub- threshold data have recovery ratios less than 1 , while at threshold $(0 \mathrm{~dB})$ masker level, 48 of 49 data have ratios less than 1 . This contrasts with recovery from 250 pulse/s masking: For sub-threshold maskers, the median ratio is close to $1(0.95)$ as is the median ratio at threshold (0.94). Although sub-threshold effects for $250 \mathrm{pulse} / \mathrm{s}$ maskers are small, a paired $t$ test across the sub-threshold data (i.e., masked vs. unmasked probe spike rates) indicates significance $(t=-3.66$, $\left.p_{\text {error }}=0.0020, d f=17\right)$. Finally, for both masker rates, median trends indicate greater probe response decrements for supra-threshold masker increments. Thus, the slopes of the functions of Figure $2 \mathrm{C}$ are greater for supra-threshold masker levels than for sub- threshold masker levels. The greater slopes may be due to the combined effects of the sub-threshold mechanism and a second contribution to membrane changes caused by action potentials.

The median cat data are replicated in Figure 2 C, $\mathrm{D}$, to provide comparisons with the computer model results, which includes data from the full model (circles) and the model without Klt channels (square symbols). With 5,000 pulse/s masking, the full model produced sub-threshold masking, but the effect was somewhat smaller than the cat median trend. However, the full model has two trends in common with the cat data. First, the decrements in the probe recovery ratio increase as masker-level increments progress through the supra-threshold range. Second, the sub-threshold effect is larger for the higher-rate masker; only a small effect was observed with lowerrate masking. Importantly, the model variant without Klt channels did not produce sub-threshold masking for either masker pulse rate, which leads us to propose the need for both the adaptation element and Klt channels in a biophysical model. The generally smaller masking effects produced by the models are likely due, in part, to the smaller duration of the model masker (200 vs. $300 \mathrm{~ms}$ ) as well as its use of higher overall probe-response FEs. The influence of the effective level of the probe (i.e., spike rate to the probe) is addressed below.

Masker-driven activity influenced probe responses differently for the two masker rates. Figure 3 plots recovery ratios for both 5,000 and 250 pulse/s masker trains across cat data as functions of the mean response rate to the masker. Plotting the ratios versus 


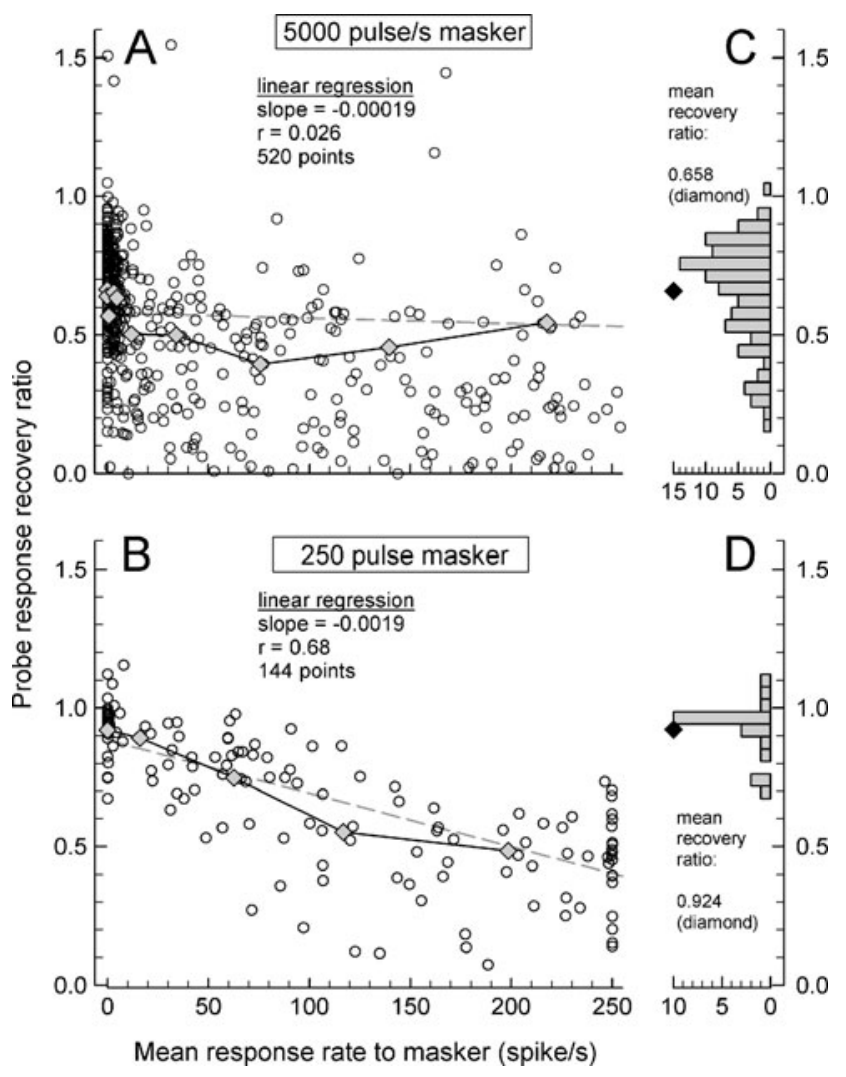

FIG. 3. The relationship between masker-evoked activity and probe-response masking differs across the two masking pulse rates used in this study. In $A$ and $B$, individual ANF recovery ratios are plotted using open circles; gray diamonds indicate medians based on groups of 50 and 24 data subsets for 5,000 and 250 pulse/s masking, respectively. Linear regressions are shown using dashed lines. The histograms of $C$ and $D$ are based upon the sub-threshold data (i.e., 0 spike/s to the masker), with mean values indicated by the black diamonds.

masker spike rates provides a measure of the influence of prior spiking; sub-threshold effects are collapsed at the point where the rate to the masker equals zero. For the 5,000-pulse/s masker, median values (diamonds in Fig. 3 A) were computed over contiguous sets of 50 observations (circles), while each median for the $250 \mathrm{pulse} / \mathrm{s}$ masker data (diamonds within Fig. 3 B) was based upon 24 observations. Different trends are seen for the two masker rates. For high-rate masking, a wide range of ratios is observed across sub-threshold levels, where median values cluster around ratios near 0.65 , reflecting a robust sub-threshold effect. Adding maskerevoked activity (i.e., supra-threshold masking) resulted in only small additional decrements, as the median supra-threshold ratios are near 0.5. This small systematic effect of masker-evoked activity is also reflected in the nearly flat linear regression. In contrast to the high-rate trends, low-rate maskers resulted in relatively little sub-threshold masking (median ratio $=0.92$ ), while masker-evoked spiking resulted in monotonically increasing degrees of masking with increases in masker activity. The histograms (Fig. $3 \mathrm{C}, \mathrm{D}$ ) summarize the sub-threshold ratios for each masker rate.

The influence of the response rates to the masker and the probe are summarized in Figure 4, where recovery ratios are plotted versus the mean response to the unmasked probe for both masker pulse rates. There is considerable scatter in recovery ratios; as could be expected, the scatter is the greatest for low response rates to the probe. The ratios tend toward higher values as the probe response rate increases. In both graphs, individual data are divided into groups (indicated by different symbols) according to the mean response rate to the masker. Line segments connect symbols that indicate median values based

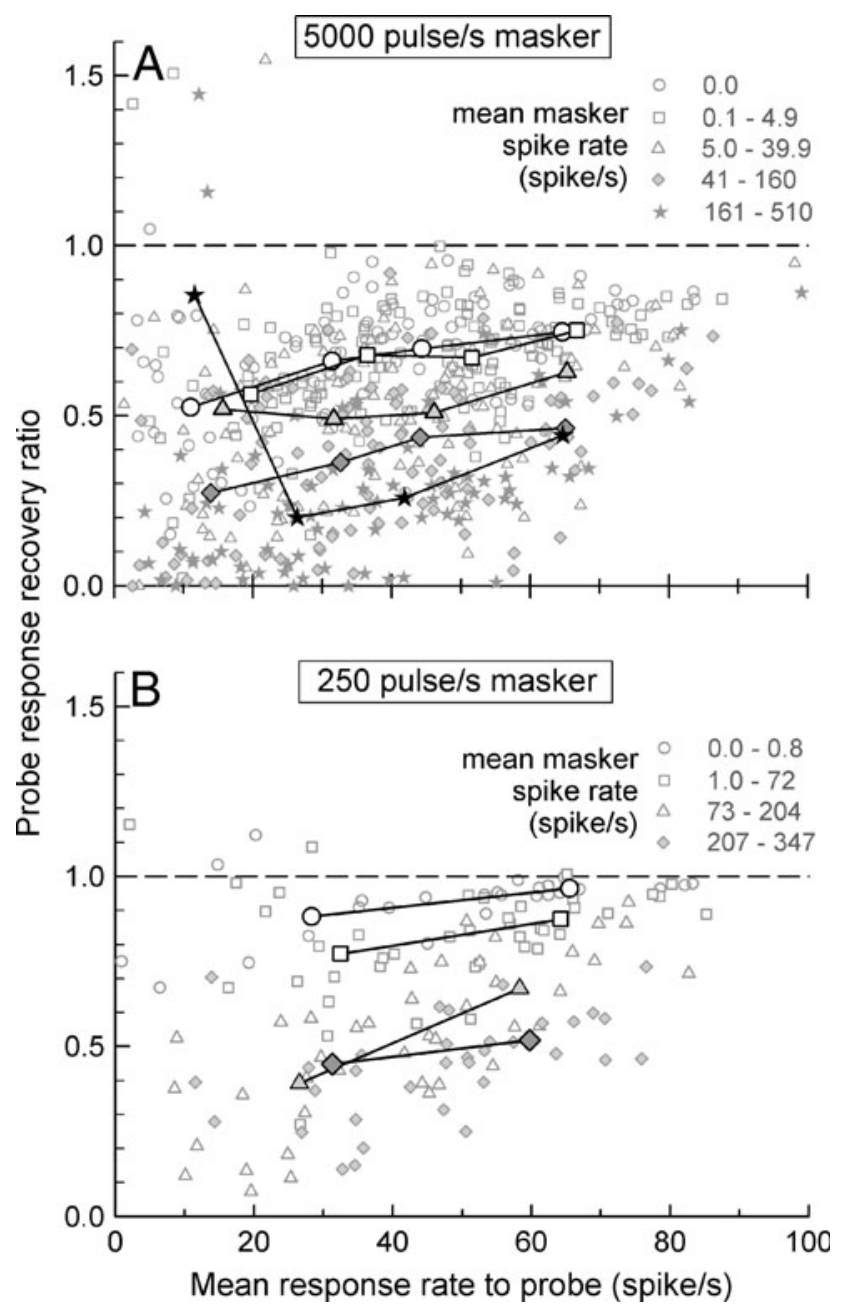

FIG. 4. Plots of recovery ratios as functions of the response rate to the probe (abscissa) and the response rate to the masker (parameter). Data from individual ANFs are plotted along with median values (symbols connected by line segments). For low probe response rates, there are relatively high degrees of scatter and indications of error (i.e., ratios $>1$ ); this is likely due to error inherent to the use of ratios of small numbers and limited sample sizes. Note that only for limited masker and probe conditions can increases in probe level overcome forward-masking rate decrements. 
(on average) on 24 observations; in one case (for the lowest masker and probe response rates with 250 pulse/s masking), the median was computed over the smallest number (14) of observations.

Generally, the median data indicate that changes in the response rate to the masker were larger than the changes that occurred by varying the probe response rate. In comparing the median recovery ratios across the two masker rates (Fig. 4 A, B), one sees similar rates of change (slopes) in the ratios across the ranges of spike rates to the unmasked probe. However, there is a masker-rate interaction. Relative to the low-rate functions (Fig. $4 \mathrm{~B}$ ), the slopes of the high-rate functions (Fig. 4 A) are typically shifted to lower ratios. In the case of low-rate masking, probe increases could essentially overcome masking effects for high probe response rates and low masker response rates. However, for other combinations of masker and probe response rates, it is not evident that probe increases can fully overcome forward-masking effects, even for low response rates to the masker.

Across the different combinations of effective masker and probe levels and for both masker pulse rates, a general trend is that a probe preceded by a masker evokes fewer spikes and that larger proberesponse decrements occur for lower unmasked probe responses. Assuming that unmasked probe responses are proportional to probe level, these trends suggest that the forward-masked rate-level functions for the masked probe would be shifted to the right (i.e., have higher thresholds) and have steeper slopes when compared to the unmasked probe rate-level functions.

\section{Effect of model variations on membrane integration}

The model comparisons of Figure 2 suggest that Klt channel activation is largely, if not solely, responsible for sub-threshold effects. Figure 5 explores model mechanisms by showing how model components varied in response to sub- and supra-threshold stimuli presented at 250 and 5,000 pulse/s. Membrane voltage, ionic currents, and $\left[K_{\text {ext }}\right]$ are plotted vs. time in each column. For both train rates, high-threshold $\mathrm{K}$ cation currents are nil for sub-threshold train levels. In contrast, low-threshold $\mathrm{K}$ currents contribute to accumulation of external $\mathrm{K}$ cations. Pulse rate clearly affects the sub-threshold changes in $\left[K_{\text {ext }}\right]$. For the $250 \mathrm{pulse} / \mathrm{s}$ train, $\left[K_{\text {ext }}\right]$ undergoes a $4.4 \%$ increase, from 5.51 to $6.17 \mathrm{mM}$ at $60 \mathrm{~ms}$ past train onset. For $5,000 \mathrm{pulse} / \mathrm{s}$ stimulation, it increases $48 \%$, from 5.51 to $10.2 \mathrm{mM}$ over $60 \mathrm{~ms}$. As $\left[K_{\text {ext }}\right]$ rises and decays in response to a transient outward current, the additivity of the "sub-threshold $\left[K_{\text {ext }}\right]$ component" can be seen as a strictly increasing function of $\left[K_{\text {ext }}\right]$ vs. time for the 5,000-pulse/s, supra-threshold, stimulus condition. Although not shown in the figure, $\left[K_{\text {ext }}\right]$ reached an asymptote within $100 \mathrm{~ms}$ of the train onset.

To further examine the hypothesis that Klt channels are required for sub-threshold masking, the model without Klt channels was run for several values of Kht channel density $\left(\rho_{\mathrm{Kht}}\right)$. Optimization of that model, to achieve acceptable approximations of physiological properties of refractoriness and rate adaptation, led to setting $\rho_{\text {Kht }}$ to 40 channels $/ \mu \mathrm{m}^{2}$. With this model, simulations were run for $\rho_{\text {Kht }}$ values of $5,10,40$, and 80 channels $/ \mu^{2}{ }^{2}$ using sub-threshold 5,000 pulse/s train stimuli within $0.28 \mathrm{~dB}$ below levels needed to evoke any action potentials. Each simulation was run for a train duration of $60 \mathrm{~ms}$. Within this time period, the full model produced a $40 \%$ increase in $\left[K_{\text {ext }}\right]$, as seen in Figure 5. Across all tested $\rho_{\text {Kht }}$ channel densities for the simpler model, none produced a change in $\left[K_{\text {ext }}\right]$ greater than our $0.01 \%$ limit of measurement precision.

\section{Effect of model channel densities}

We sought to understand how the relative nodal densities of Klt and Kht channels influence key temporal response properties of the model. Figure 6 summarizes the results of variations on the full model in which the relative ratio of Klt and Kht channel densities was manipulated from 0:1 (i.e., the Kht-only model) to a ratio as high as 4:1. Figure $6 \mathrm{~A}$ shows four PSTHs, one obtained from a typical cat ANF and three obtained using models with different ratios of $\mathrm{K}$ cation channel densities (i.e., $\rho_{\mathrm{Klt}} / \rho_{\mathrm{Kht}}$ set to $1: 1,2: 1$, and $4: 1$, as indicated in the plots). In all cases, a 100ms-long 5,000-pulse/s train of biphasic pulses was used. Relative to the chosen exemplar cat ANF PSTHs, the PSTHs of the models indicate that rate adaptation increases as the $\rho_{\mathrm{Klt}} / \rho_{\mathrm{Kht}}$ ratio is increased.

A quantitative comparison of cat and model spikerate adaptation is provided by Figure $6 \mathrm{~B}$. PSTHs to $5,000 \mathrm{pulse} / \mathrm{s}$ trains were fit by a single negativeexponent decaying function to provide the adaptation time constant, $\tau$. The cat ANF rate-adaptation time constants were taken from Zhang et al. (2007, Fig. 7) and replotted over the range of onset firing rates (i.e., rates over the first $12 \mathrm{~ms}$ ) corresponding to the present model simulations. The stimulus levels used for the modeling were chosen so that the onset rates were within the range of the cat data. The resulting $\tau$ 's for the model simulations using a range of $\rho_{\mathrm{Klt}} / \rho_{\mathrm{Kht}}$ ratios are also plotted in Figure $6 \mathrm{~B}$, where it is apparent that smaller ratios result in closer approximations to the cat data.

Another key temporal measure is the probe response recovery ratio, as it assesses the restoration of the neuron from prior adaptation and may involve 


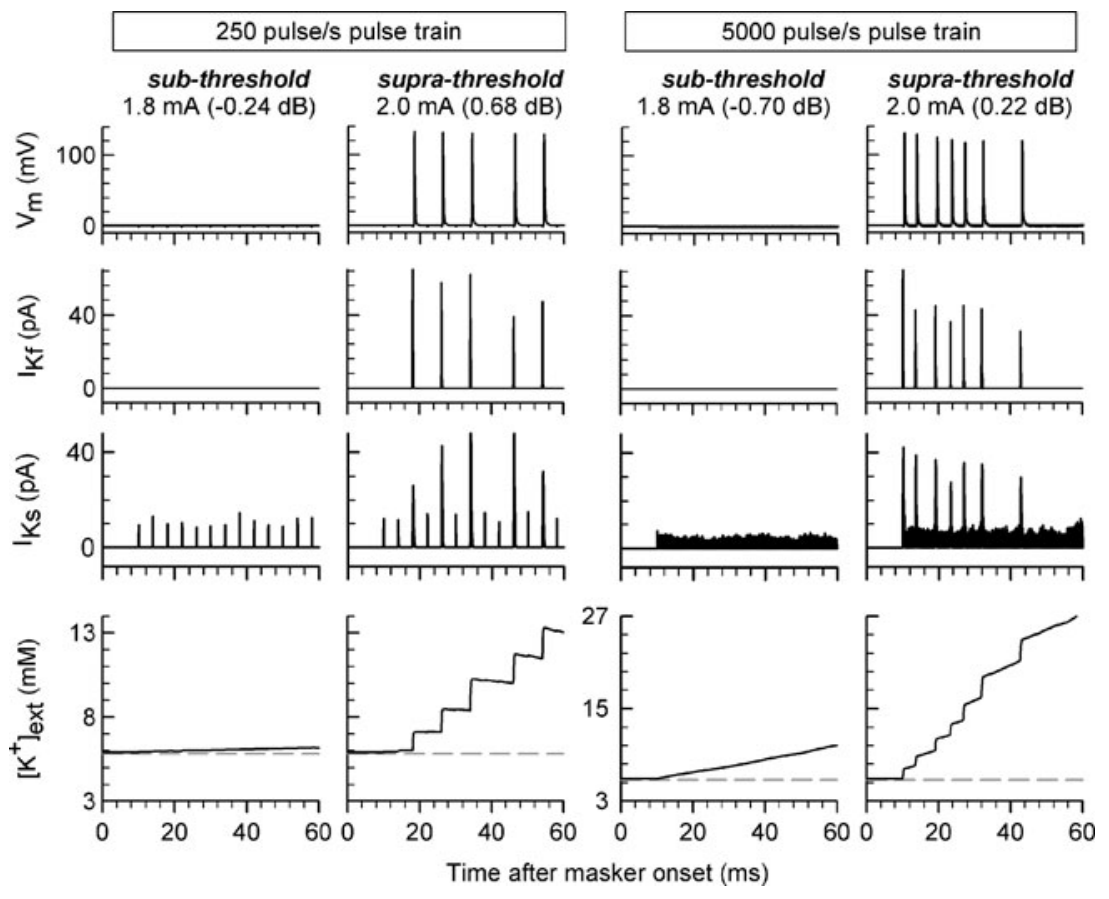

FIG. 5. Biophysical model responses to pulse train stimuli, showing how membrane voltage $\left(V_{\mathrm{m}}\right)$ Kht and Klt ion currents ( $I_{\mathrm{Kf}}$ and $I_{\mathrm{Ks}}$, respectively) and extracelluar $K$ concentration $\left(\left[K_{\text {ext }}\right]\right)$ vary across stimulus presentation time. Currents through Klt channels result in accumulation of $\left[K_{\text {ext }}\right]$, as does spike activity for the case of 5,000 pulse/s stimuli. Increase in $\left[K_{\text {ext }}\right]$ is barely visible for the case of sub-threshold, 250 pulse/s stimuli. Dashed lines indicate the quiescent level of $\left[K_{\text {ext }}\right]$. The abscissae indicate time after the onset of each pulse train.

different membrane mechanisms than those active during rate adaptation. Figure $6 \mathrm{C}$ shows recovery ratios for the four $\rho_{\mathrm{Klt}} / \rho_{\mathrm{Kht}}$ ratios, along with a statistical summary of feline data obtained in this study. For both the feline and model data, the masker level was chosen to be at or near threshold and the probe level was adjusted to obtain firing probabilities between 0.8 and 0.9 . In this case, the model provided better approximations of cat-like recovery ratios when the two highest (2:1 and 4:1) K channel densities were used. The recovery ratio for the 1:1 density ratio was within 1 standard deviation of the mean feline data. Finally, a two-pulse (masker-probe) paradigm was used to determine the absolute refractory period (ARP) for the model variations, comparing those data against the cat ARPs reported in Miller et al. (2001b). This comparison is shown in Figure 6D. In this case, of the three density ratios with nonzero $\rho_{\mathrm{Kl}}$, the $1: 1$ ratio was slightly better than the higher ratios, although all simulations show a bias toward higher ARPs in comparison to the cat data. Taken together, these analyses of the effect of the $\rho_{\mathrm{Klt}} / \rho_{\mathrm{Kht}}$ ratio not only indicate why we chose the 1:1 ratio but also indicate that any specific ratio is a compromise, as no one ratio fits best to the cat ANF response properties shown in Figure 6.

\section{DISCUSSION}

This report shows that electric stimulation of feline and modeled ANFs can result in the forward masking of subsequent stimuli, even for masker levels set below the level required to evoke any response (i.e., spikes) to the masker train. Decreased responses to the masked probe (relative to the unmasked probe response) were observed over the entire range of probe levels examined, although the decrements were generally smaller for greater effective probe levels. The two trends are observed in the summary data of Figure 4 and suggest that, relative to unmasked ratelevel data, the masked probe rate-level functions would be expected to shift to higher probe levels and have a modest increase in slope. Data from both cat and simulated ANFs indicate that the sub-threshold masking effects are much greater for the 5,000pulse/s masker than for the slower 250-pulse/s masker (Fig. 2). The degree of forward masking was also examined as a function of the degree of spike activity evoked by the masker train and the unmasked probe train (Fig. 4). At least over the ranges of levels explored in this study, greater increases in masking were caused by masker-level increases than by decreases in probe level. Sub-threshold masking could be observed over a wide range of masker levels. Group trends suggest that with 5,000 pulse/s masking, subthreshold effects were evident over at least a 4-dB range of masker levels. This is significant, as ANF ratelevel functions for single-pulse stimulation typically have 1- to 2-dB dynamic ranges (Miller et al. 1999, 2001a).

Sub-threshold masking has implications for the design of cochlear prostheses, as well as clinical prostheses for functional electrical stimulation of other neural systems. It also indicates the importance for continued development of more realistic computational models of nerve fibers. A conventional view of rate adaptation is that masker-evoked spike activity 

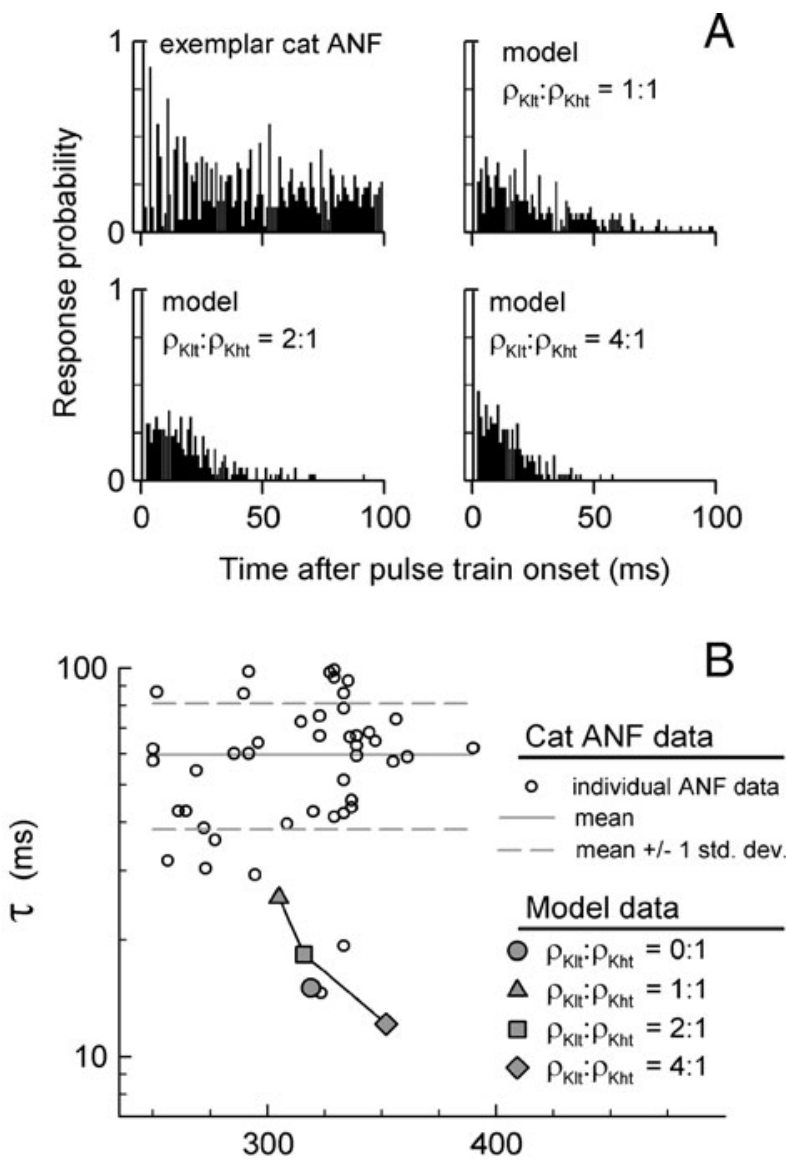

Onset response rate (spike/s)

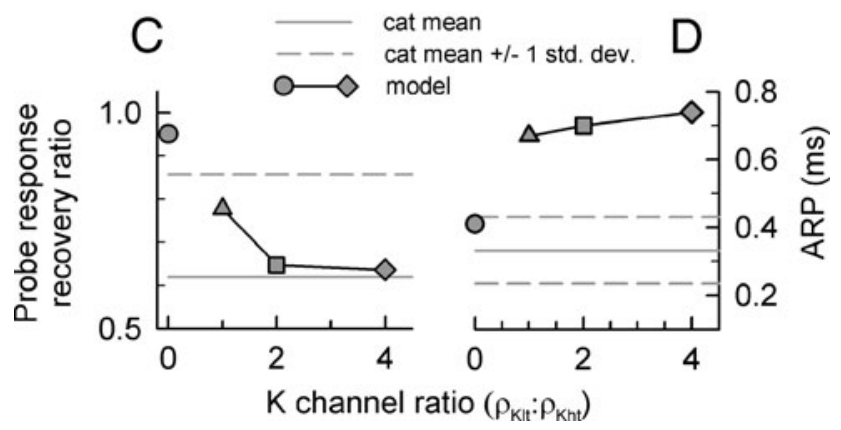

reduces the response of membrane elements or ionic stores. The comparisons of computer model simulations show that the addition of Klt channels to a model that provides $K_{\text {ext }}$ accumulation (Woo et al. 2009a, b) results in much larger sub-threshold masking that what was observed without Klt channels (Fig. 2 C). Furthermore, the magnitude of subthreshold forward masking is strongly dependent upon masker pulse rate (Fig. 2), with the higher masker rate producing a larger $\left[K_{\text {ext }}\right]$ increment (Fig. 5). The ANF simulations suggest that the model element responsible for sub-threshold masking is the Klt channel. Certainly its addition greatly increased sub-threshold changes in $\left[K_{\text {ext }}\right]$ in a manner consistent
4 FIG. 6. Variations in the ratio of Klt and Kht channels influence the model's predictions for rate adaptation (A, B), the recovery of ANFs to prior masking $(\mathbf{C})$, and the ARP (D). A PSTH histograms from a selected cat ANF (upper left) and variations in the full model where different $\rho_{\mathrm{KI}} / \rho_{\mathrm{Kht}}$ ratios were explored. The cat PSTH was chosen as it was representative of ANF responses to 5,000 pulse/s trains that elicited a strong onset spike rate. The three model results in $\mathbf{A}$ all exhibit strong degrees of rate adaptation. Rate adaptation to $5,000 \mathrm{pulse} / \mathrm{s}$ trains for cat ANFs and model simulations are compared in B by plotting adaptation time constants for cat ANFs (small circles) and for four model variations that explored different $\rho_{\mathrm{Kl}} / \rho_{\text {Kht }}$ ratios. The mean and 1 standard deviation from the mean are plotted as horizontal lines (solid line: mean; dashed lines: \pm 1 standard deviation from the mean). In $\mathbf{B}$, the cat ANF data were derived from Zhang et al. (2007). In C, the probe response recovery ratio (see text) for different model $\rho_{\mathrm{KI}} / \rho_{\mathrm{Kht}}$ ratios are compared with the mean value \pm 1 standard deviation of cat data obtained in the present study. Similar cat vs. model comparisons are provided for the ARP in $\mathbf{D}$. In $\mathbf{B}-\mathbf{D}$, the same filled symbol types refer to the $\rho_{\mathrm{KI}} / \rho_{\mathrm{Kht}}$ ratios shown in $\mathbf{B}$.

with masking (Fig. 5). However, while the relatively simple combination of the integration of $K_{\text {ext }}$ current and Klt channels has a degree of face validity, our results are incapable of proving the assertion that Klt channels are essential to the observed sub-threshold effects.

An important distinction related to electric excitation of ANFs is that direct, sub-threshold, electrical stimulation of membranes (such as that which occurs with prosthetic stimulation) may be predicted to alter ion channel responses and result in cumulative changes to the membrane, whereas such effects may not be expected with sub-threshold acoustic stimulation of the normal auditory periphery. It is well established that supra-threshold excitation of ANFs results in responses that differ significantly in several ways from those obtained with acoustic stimulation of the cochlea. As suggested from the trends of Figure 3, the rate-level functions for forward-masked electric probe trains would be expected to be shifted to higher levels, i.e., have relatively higher thresholds.

We note that no experimental procedure used to deafen all ANFs is likely to be completely successful; it is possible that we recorded from some fibers associated with a surviving and viable inner hair cell. Yet, our Neomycin protocol required a minimum 80$\mathrm{dB}$ loss of sensitivity as assessed by the ABR. Furthermore, no fibers used in this study demonstrated electrophonic effects. In contrast, the sub-threshold effect was broadly observed, as indicated by the groupdata plots (Figs. 2, 3, and 4). We conclude that acoustic sensitivity was not required for the subthreshold effect.

A possible concern is the use of high-rate, longduration pulse trains to excite ANFs. While we kept all stimuli well below levels that can cause irreversible hydrolysis to occur over one phase of a biphasic pulse (Brummer and Turner 1977), the question of possible 
damage induced by high-rate pulse trains may warrant additional research. Such stimuli may, through nonlinear reactions other than that described by Brummer and Turner (either by ANFs themselves or through some failure of prosthetic hardware to block all d.c. components of a stimulus), result in functional alteration of ANFs. Xu et al. (1997) examined changes in the electrically evoked auditory brainstem response (EABR) and cochlear histology in cats chronically implanted and stimulated with trains of pulse rates as high as 2,000 pulse/s. Levels were kept lower than the relatively conservative $\left(\sim 30 \mu \mathrm{C} / \mathrm{cm}^{2}\right)$ current densities suggested as safe for chronic stimulation (Walsh and LeakeJones 1982; Leake et al. 1990). While histological analyses suggested that 2,000-pulse/s chronic stimulation was safe, some elevation of EABR thresholds was reported by Xu et al. (1997). A systematic analysis of short-term and chronic effects of high-rate pulse-train stimulation may be warranted, particularly given the possible use of pulse rates of 5,000 pulse/s or higher.

The computational simulations reinforce the cat ANF findings and suggest that sub-threshold phenomena are due to membrane effects that contribute to altered Nernst potentials and activation thresholds. The model with low- and high-threshold $\mathrm{K}$ channels exhibited the sub-threshold effect, while model variants without Klt channels (i.e., models with only Kht channels) did not. There are several classes of ANF $\mathrm{K}$ channels with different sensitivities and kinetics (Davis 1996; Adamson et al. 2002; Mo and Davis 1997). Traditionally, ANF models have only included Kht channels and only recently have computational models of ANFs included Klt channels, as they improve predictions of spike-rate adaptation (Negm and Bruce 2008; Imennov and Rubinstein 2009). In a model of mammalian motoneurons (McIntyre et al. 2002), Klt channels were found to be the primary determinant of the afterhyperpolarization phase, in contrast to the relatively small influence of Kht channels.

Interpretation of our model results requires some caution, as our parametric analysis of the $\rho_{\mathrm{Klt}} / \rho_{\mathrm{Kht}}$ ratio changes suggests that the model is not capable of providing optimal solutions for rate adaptation, adaptation recovery, and ARP (Fig. 6). Also, across our model simulations, we did not observe the short-term enhancement of probe responses attributed to the integration of masker energy (Fig. 1). Thus, while the model successfully demonstrated sub-threshold masking, it is likely a simplification of membrane gate electrophysiology of cat ANFs. One difficulty with modeling is the desire for a parsimonious solution and also accurate results across several response domains. We urge caution at this point because other work, for example, indicates that the curvature of the fiber-hence the distribution of the excitation fieldinfluences modeled ANF responses (Woo et al. 2010).
We suggest that the inclusion of additional channels in new models not be done at the expense of overlooking (1) fiber diameter variations, (2) the complex paths of ANFs through space, and (3) the use of finite-element approaches to better predict the spatial spread of electric currents.

Other mechanisms, not explored here, may contribute to adaptation or sub-threshold responses. Voltage-dependent $\mathrm{Ca}^{++}$-gated channels can affect rate adaptation by gating Ca-dependent $\mathrm{Na}$ or $\mathrm{K}$ channels (Schwindt et al. 1989; Sah 1996), and other iondependent gates affecting firing rate have been identified (Bhattacharjee and Kaczmarek 2005). There is also evidence that ANFs contain multiple classes of $\mathrm{K}$ channels that can be differentially distributed along the apical-to-base cochlear dimension (Mo and Davis 1997; Adamson et al. 2002; Liu and Davis 2007). It has been suggested that unique cochleotopic distributions of $\mathrm{K}$ channel sub-types may be consistent with other cochleotopic properties that influence stimulus coding by the population of ANFs (Adamson et al. 2002). While our relatively simple model with two $\mathrm{K}$ channel types provides a parsimonious mechanism to our cat data, it is clear that there is much room for improvement in the computer modeling of ANF populations excited by intracochlear stimulation.

While some mechanisms of forward masking may be presumed to arise from central processing, this paper describes a novel effect at the peripheral, ANF level of the auditory system. This may have implications for future studies of masking and attempts to attribute masking at a particular afferent nucleus. Possible future work could include attempts to assess sub-threshold masking using ECAP or EABR measures accessible to clinical research of human populations, although it may be relatively difficult to observe the effect from the gross, or fiber-population, measures. Psychophysical measures of sub-threshold masking could also be proposed. It would be interesting to determine whether or not this ANF phenomenon is reflected or lost in centrally mediated responses.

Sub-threshold masking may necessitate re-evaluation of clinical practices and prosthesis designs to optimize their effectiveness for clinical populations. For ANFs, electric current from an intracochlear electrode is assumed to excite a population of fibers that respond with action potentials. Our data suggest that an electrode also exerts a sub-threshold influence on a second fiber population that presumably extends further across space. A simple conceptualization of this second ANF population is that it flanks, or surrounds, the excited population. This assumption may be reasonable for a linear array of fibers but may be too simplistic given the spiraling configuration of cochlear fibers, which has been modeled and shown 
to produce somewhat more complicated spatial patterns of activation (Frijns et al. 2001).

This report improves our understanding of how prostheses with multiple, spatially segregated, channels of electric stimulation may alter neural states. It could be conjectured that monopolar stimulus fields (i.e., fields with relatively small changes across space) may result in more widespread sub-threshold effects than would more spatially restricted fields. We can no longer assume that stimulus-channel interactions are limited to the refractory effects caused by one channel on the excitation region of a second channel or that sub-threshold channel interactions only involve temporal integration or simultaneous effects. Our data indicate that sub-threshold effects can persist over a range of sub-threshold stimuli as great as $8 \mathrm{~dB}$, suggesting that a potentially broad spatial region of sub-threshold effects has not been examined or accounted for in the design of prosthetic stimulation devices. Thus, measures of sub-threshold masking could be proposed as another aspect of peripherally based (i.e., ECAP-based) or centrally based (i.e., psychophysical) channel interaction measures.

It is not possible to assert, from the data collected in this study, whether or not sub-threshold effects present another constraint on prosthetic excitation or could be used in some manner to enhance spatial selectivity. This limitation is due in part to our simple modeling of extra-neural tissue and the possibility that additional nodal channels require inclusion. Thus, follow-up studies done in animal models and clinical settings should approach this phenomenon in ways not strictly limited to our stimulus conditions.

\section{ACKNOWLEDGMENTS}

This work was funded by NIH grant R01-DC006478. The authors would like to acknowledge the helpful comments of Dr. Kevin Kilgore of Case-Western University and the Cleveland FES Center regarding nonauditory computational models. We also wish to acknowledge our consultants, Chris van den Honert and David Ryugo, for their assistance with grant-related research.

\section{REFERENCES}

Adamson CL, Reid MA, Mo ZL, Bowne-English J, Davis RL (2002) Firing FEATURES AND POTASSIUM CHANNEL CONTENT OF MURINE SPIRAL GANGLION NEURONS VARY WITH COCHLEAR LOCATION. J Comp Neurol 447:331-350

Arnesen AR, Osen KK (1978) The Cochlear nerve in the cat: TOPOGRAPHY, COCHLEOTOPY, AND FIBER SPECTRUM. J Comp Neurol 178:661-678

Bal R, Ortel D (2001) Potassium currents in octopus cells of the Mammalian COCHLEAR NuCleus. J Neurophys 86:2299-2311
Bhattacharjee A, Kaczmarek LK (2005) For K+ channels, Na+ is the NEW CA2+. Trends Neurosci 28:422-428

Brew HM, Forsythe ID (1995) Two voltage-Dependent K+ CONduc TANCES WITH COMPLEMENTARY FUNCTIONS IN POST-SYNAPTIC INTEGRATION AT A CENTRAL AUDITORY SYNAPSE. J Neurosci 15:8011-8022

Bruce I, Negm M (2009) IKLT and Ih may EXPlain Rapid and SHORTTERM SPIKE-RATE ADAPTATION IN AUDITORY NERVE FIBER RESPONSES TO COChlear implant stimulation. Assoc Res Otolaryngol Abs p 327

Brummer SB, Turner MJ (1977) Electrical stimulation with Pt ELECTRODES: II-ESTIMATION OF MAXIMUM SURFACE REDOX (THEORETICAL NON-GASSING) LIMITs. IEEE Trans Biomed Eng 24:440-443

Cartee LA, van den Honert C, Finley CC, Miller RL (2000) EVALUATION OF A MODEL OF THE COCHLEAR NEURAL MEMBRANE. I. Physiological measurement of membrane characteristics in response to intrameatal electrical stimulation. Hear Res 146:143-152

Chow CC, White JA (1996) Spontaneous action potentials due to Channel fluctuations. Biophys J 71:3013-3021

Colombo J, Parkins CW (1987) A model of electrical excitation of THE MAMMALIAN AUdITORz'NERVE NEURON. Hear Res 31:287-311

Davis RL (1996) Differential distribution of potassium ChanNels IN ACUTELY DEMYELINATED, PRIMARYAAUDITORY NEURONS IN VITRO. J NeUROphysiol 76:438-447

DyNes SBC (1996) DisCHARGE CHARACTERISTICS OF AUdITORY NERVE FIBERS fOR pUlsatile electrical stimuli. Ph.D. thesis, Massachusetts Institute of Technology, p 125 leaves

Frijns JH, Briaire JJ, Grote JJ (2001) The importance of human COCHLEAR ANATOMY FOR THE RESULTS OF MODIOLUS-HUGGING MULTICHANNEL COCHLEAR IMPLANTS. Otol Neurotol 22:340-349

Hartmann R, Topp G, Kuinke R (1984) Discharge patterns of cat PRIMARY AUDITORY FIBERS WITH ELECTRICAL STIMULATION OF THE COCHLEA. Hear Res 13:47-62

ImenNov NS, Rubinstein JT (2009) Stochastic population model For ELECTRICAL STIMULATION OF THE AUDITORY NERVE. IEEE Trans Biomed Eng 56:2493-2501

JaVEl E (1990) ACOUSTIC AND ELECTRICAL ENCODING OF TEMPORAL INFORMATION. In: Miller JM, Spelman FA (eds) Cochlear implants. Models of the electrically stimulated ear. Springer, New York, pp 247-295

Krylov BV, Makovsky VS (1978) SPIKe frequency aDaptation in AMPHIBIAN SENSORY FIBRES IS PROBABLY DUE TO LOW-THRESHOLD K CHANNELS. Nature 275:549-551

Leake PA, Kessler DK, Merzenich MM (1990) Application and safety OF COChlear prostheses. In: Agnew WF, McCreery DB (eds) Neural prostheses: fundamental studies. Prentice Hall, Englewood Cliffs, pp 253-296

Liberman MC, Oliver ME (1984) Morphometry of intracellularly LABELED NEURONS OF THE AUDITORY NERVE: CORRELATIONS WITH FUNCTIONAL PROPERTIES. J Comp Neurol 223:163-176

Litvak LM, Smith ZM, Delgutte B, Eddington DK (2003) DesynchRONIZATION OF ELECTRICALLY EVOKED AUDITORY-NERVE ACTIVITY BY HIGHFREQUENCY PUlSE tRAINS OF LONG DURATION. J Acoust Soc Am 114:2066-2078

Liu Q Davis RL (2007) Regional specification of threshold SENSITIVITY AND RESPONSE TIME IN CBA/CAJ MOUSE SPIRAL GANGLION NEurons. J Neurophysiol 98:2215-2222

Matsuoka AJ, Rubinstein JT, Abbas PJ, Miller CA (2001) The effects OF INTERPULSE INTERVAL ON STOCHASTIC PROPERTIES OF ELECTRICAL STIMULATION: MODELS AND MEASUREMENTS. IEEE Trans Biomed Eng $48: 416-424$

McIntyre CC, Richardson AG, Grill WM (2002) Modeling the EXCITABILITY OF MAMMALIAN NERVE FIBERS: INFLUENCE OF AFTERPOTENTIALS ON THE RECOVERY CYCLE. J Neurophysiol 87:995-1006

McNeAl DR (1976) ANALYsis of a MODEL FOR EXCITATION OF MYELINATED NERVE. IEEE Trans Biomed Eng 23:329-337

Miller CA, Abbas PJ, Robinson BK, Rubinstein JT, Matsuoka AJ (1999) Electrically EVOKed SINGLE-Fiber action pOteNTIALS From 
CAT: RESPONSES TO MONOPOLAR, MONOPHASIC STIMULATION. Hear Res 130:197-218

Miller CA, Robinson BK, Rubinstein JT, Abbas PJ, Runge-Samuelson CL (2001A) AUdITORY NERVE RESPONSES TO MONOPHASIC AND BIPHASIC ELECTRIC STIMUli. Hear Res 151:79-94

Miller CA, Abbas PJ, Robinson BK (2001в) Response properties of THE REFRACTORY AUDITORY NERVE FIBER. J Assoc Res Otolaryngol 2:216-232

Miller CA, Hu N, Zhang F, Robinson BK, Abbas PJ (2008) Changes ACROSS TIME IN THE TEMPORAL RESPONSES OF AUDITORY NERVE FIBERS Stimulated by Electric pulse trains. J Assoc Res Otolaryngol 9:122-137

Miller CA, Robinson BK, Hetke JF, Abbas PJ, Nourski KV (2004) FEASIBILITY OF USING SILICON-SUBSTRATE RECORDING ELECTRODES WITHIN THE AUDITORY NERVE. Hear Res 198:48-58

Mino H, Rubinstein JT, Miller CA, Abbas PJ (2004) Effects of ELECTRODE-TO-FIBER DISTANCE ON TEMPORAL NEURAL RESPONSE with electrical stimulation. IEEE Trans Biomed Eng $51: 13-20$

Mino H, Rubinstein JT, White JA (2002) Comparison of algorithms FOR THE SIMULATION OF ACTION POTENTIALS WITH STOCHASTIC SODIUM CHANNELs. Ann Biomed Eng 30:578-587

Mo Z-L, Davis RL (1997) ENDOgENous Firing PATterns of MURINE SPIRAL GANGLION NEURONS. J Neurophysiol 77:1294-1305

Negm MH, Bruce IC (2008) Effects of I(H) and I(KLT) on the RESPONSE OF THE AUDITORY NERVE TO ELECTRICAL STIMULATION IN A stochastic Hodgkin-Huxley model. Conf Proc IEEE Eng Med Biol Soc 2008:5539-5542

SAH P (1996) $\mathrm{CA}^{2+}$-activated $\mathrm{K}^{+}$currents in neurones: types, physiological roles and modulation. Trends Neurosci 19:10154
Schwarz JR, EikHof G (1987) Na CURRENTS AND ACtion POtentials IN RAT MYELINATED NERVE FIBRES AT 20 AND $37^{\circ}$ C. Pflügers Arch 409:569-577

Schwarz JR, Reid G, Bostock H (1995) Action potentials and Membrane CURRENTS IN THE human NOde OF RANVIER. Pflügers Arch 430:283-292

Schwindt PC, Spain WJ, Crill WE (1989) Long-Lasting reduction of EXCITABILITY BY A SODIUM-DEPENDENT POTASSIUM CURRENT IN CAT NEOCORTICAL NEURONS. J Neurophysiol 61:233-244

Stypulkowski PH, van den Honert C (1984) Physiological properties of the electrically stimulated auditory nerve. I. Compound action potential recordings. Hear Res 14:205-223

Walsh SM, Leake-Jones PA (1982) Chronic electrical stimulation of AUDITORY NERVE IN CAT: PHYSIOLOGICAL AND HISTOLOGICAL RESUlTS. Hear Res 7:281-304

Woo J, Miller CA, Abbas PJ (2009a) Biophysical model of an auditory NERVE FIBER WITH A NOVEL ADAPTATION COMPONENT. IEEE Trans Biomed Eng 56:2177-2180

Woo J, Miller CA, Abbas PJ (2009в) Simulation of the electrically STIMULATED COCHLEAR NEURON: MODELING ADAPTATION TO TRAINS OF ELECTRIC PULSES. IEEE Trans Biomed Eng 56:1348-1359

Woo J, Miller CA, Abbas PJ (2010) The dependence of Auditory nerve RATE ADAPTATION ON ELECTRIC STIMUlus parameters, ELECTROde POSITION, AND FIBER DIAMETER: A COMPUTER MODEL STUdy. J Assoc Res Otolaryngol 11:283-296

Xu J, Shepherd RK, Millard RE, Clark GM (1997) Chronic electrical STIMULATION OF THE AUditORY NERVE AT HIGH STIMULUS RATES: A PHYSIOLOGICAL AND HISTOPATHOLOGICAL STUDY. Hear Res 105:1-29

Zhang F, Miller CA, Robinson BK, Abbas PJ, Hu N (2007) Changes ACROSS TIME IN SPIKE RATE AND SPIKE AMPLITUDE OF AUDITORY NERVE FIBERS STIMULATED BY ELECTRIC PULSE TRAINS. J Assoc Res Otolaryngol 8:356-372 